\title{
Correction: Molecular collisions coming into focus
}

Cite this: Phys. Chem. Chem. Phys., 2015, 17, 12365

\author{
Jolijn Onvlee, Sjoerd N. Vogels, Alexander von Zastrow, David H. Parker and \\ Sebastiaan Y. T. van de Meerakker*
}

DOI: $10.1039 / c 5 c p 90062 j$

Correction for 'Molecular collisions coming into focus' by Jolijn Onvlee et al., Phys. Chem. Chem. Phys., 2014, 16, 15768-15779.

www.rsc.org/pccp

Some numbers that are given on page 15771 do not correspond to the velocity distributions shown in Fig. 4 . In the third paragraph of this page it should read "The distribution has a width (FWHM) of $4.8 \mathrm{~m} \mathrm{~s}^{-1}$ and $1.4 \mathrm{~m} \mathrm{~s}^{-1}$ in the longitudinal and transverse direction, respectively. The velocity distribution can also be expressed as a speed ratio of $S=235$ (or a temperature of $T=15.0 \mathrm{mK}$ ) and a divergence of $0.17^{\circ}$ (FWHM) for the packet of NO molecules."

The Royal Society of Chemistry apologises for these errors and any consequent inconvenience to authors and readers. 